\title{
Computer therapy combined with non-invasive brain stimulation for sentence processing difficulties in post-stroke aphasia: a randomised control trial (the UTILISE study)
}

Rosemary Varley ${ }^{1,}{ }^{*}$, Claudia Bruns ${ }^{1}$, Jane Warren ${ }^{1}$, Ewa Dąbrowska ${ }^{2}$, Amir-Homayoun Javadi ${ }^{1,3,4}$

${ }^{1}$ Language \& Cognition, Psychology \& Language Sciences, University College London, UK

${ }^{2}$ Department of English and American Studies, FAU Erlangen and Department of English Language and Linguistics, University of Birmingham, UK

${ }^{3}$ School of Psychology, University of Kent, UK

${ }^{4}$ School of Rehabilitation, Tehran University of Medical Sciences, Iran

* Corresponding Author:

Email: rosemary.varley@ucl.ac.uk

Website: https://www.cognitionandgrammar.net/

Address: 2 Wakefield Street, Chandler House, London, WC1N 1PF, UK

Trial sponsor: University College London. The sponsor and funder have no role in the design, management, implementation and reporting of the study.

Trial registration: Prospectively registered on 13/09/2019 at ISRCTN14466044 https://doi.org/10.1186/ISRCTN14466044

Protocol amendments: V2 (12.11.2019); V3 (14.01.2020); V4 (06.03.2020)

Funding: The study is funded by a project grant from the UK Stroke Association (TSA SA PG 18\100054)

External Chair Steering Committee: Dr Paul Conroy (University of Manchester, UK) 


\begin{abstract}
Background: We explore the efficacy of a new computer therapy for sentence comprehension and production impairments in post-stroke aphasia. The intervention is based upon the theoretical framework of usage-based Construction Grammar, which has yet to be systematically applied to the management of sentence processing disorders in aphasia. Components of the intervention were trialled in two small case series, with the results of one pilot used to inform a power calculation. The aims of the study are: (1) To determine if UTILISE therapy (Unification Therapy Integrating LexIcon and SEntences) for aphasic sentence processing impairment is more effective than usual care; (2) To determine if non-invasive transcranial direct current stimulation (tDCS) enhances treatment outcomes; (3) To determine if improvements are evident in functional use of language after treatment; (4) To determine if any treatment effects are apparent after an 8-week maintenance period.
\end{abstract}

Methods: We will conduct a Phase II prospective randomised control trial. The behavioural component of the study (computer therapy) is single-blinded on the primary outcome measure, while the tDCS element is double-blinded. The trial is conducted within a university setting and aims to recruit 66 participants with post-stroke aphasia from community and post-acute rehabilitation settings. After first baseline evaluation, participants are randomised on a 2:2:1:1 basis to one of four conditions: 1 ) immediate treatment + active tDCS; 2 ) immediate treatment + sham tDCS; 3 ) deferred treatment + active tDCS; 4) deferred treatment + sham tDCS. A second baseline assessment is completed prior to start of treatment with a gap of four weeks between baselines for the immediate groups, and eight weeks for the deferred groups. The deferred trial entry conditions act as a usual care control across their extended baseline phase. Treatment is administered in $12 \times 60$-minute sessions over a four-week period. Outcomes are assessed immediately at the end of intervention and after an eight week no treatment period. Participants will also undergo a structural magnetic resonance (MR) brain scan to determine lesion location and extent. The primary outcome measure is degree of connectivity (ratio of three-word combinations to total number of words) in spontaneous narrative speech (personal narratives and narration of picture cartoon series). Speech samples will be audio-recorded and subsequently transcribed and tagged by raters blind to allocation and phase. Analysis will be performed by automated analysis software (Frequency in Language Analysis Tool, FLAT; Zimmerer, Newman, Thomson, Coleman, \& Varley, 2018). Secondary outcome measures are spoken sentence comprehension (Test of Reception of Grammar, TROG-2; Bishop, 2003), a studyspecific story completion test (adapted from Goodglass, Gleason, Ackerman Bernholtz, \& Hyde, 1972), and the Stroke and Aphasia Quality of Life Scale (SAQOL-39; Hilari, Byng, Lamping, \& Smith, 
2003). A shortened form of a written synonym judgement task (adapted from the Action for Dysphasic Adults Auditory Comprehension Battery, A.D.A.; Franklin, Turner, \& Ellis, 1992) is administered as a probe of an untreated behaviour.

Discussion: In a series of planned comparisons, we will first determine the stability of behaviour between the two baseline evaluations. We will examine the efficacy of UTILISE through comparison of change in scores between the immediate and deferred entry group, and subsequent maintenance of any behavioural gains by change between the immediate outcome and the follow-up maintenance measures. We will explore the effect of active vs. sham tDCS, and the relationship between outcomes and demographic and behavioural profiles, and lesion size and location.

Key words: aphasia, sentence, comprehension, production, therapy, tDCS, computer, Construction Grammar 


\section{Background}

In the UK, there are over 100,000 cases of stroke per year and 1.2 million stroke survivors (Stroke Association, 2018). A common consequence of stroke is language impairment (aphasia), which disrupts language comprehension and production. Aphasia is present in $21-38 \%$ of acute stroke cases (Berthier, 2005). As spoken and written language play a central role in human interactions, language impairment limits capacity to exchange thoughts and feelings, access print and digital media, and participate in work and lifelong education, with profound consequences for the individual and their family (Ross \& Wertz, 2003). Aphasic impairment traditionally has been addressed through the dichotomy of lexicon and grammar, based on the observation that some individuals appear to have disproportionate disruption in one or other of these language subdomains. However, most people with aphasia have both vocabulary and grammatical impairments, often spanning both comprehension and production. Although treatment for aphasia has been shown to be effective (Brady, Kelly, Godwin, Enderby, \& Campbell, 2016), the majority of impairment-level interventions have focused on the single-word level, and particularly on noun retrieval in picture naming. A typical outcome of these trials is that treated vocabulary items improve, but generalisation to untreated words or to connected speech is limited (e.g., Palmer et al., 2019). Lexical treatment directed at verbs and their associated arguments appears to have greater impact on connected speech (Edmonds, 2016; Webster, Whitworth, \& Morris, 2015). However, in comparison to single-word interventions, fewer studies have explored sentence-level therapies and those that do typically focus on the sub-group of speakers with agrammatic aphasia who display dramatic reductions in grammatical complexity (e.g., Thompson, Choy, Holland, \& Cole, 2010).

A range of techniques are employed to facilitate sentence-level processing in aphasia and the diversity of therapeutic approaches reflects a lack of consensus on the mechanisms involved in sentence processing. One group of therapies is more narrowly 'syntactic', based on proposals that core syntactic representations and computations are disrupted, particularly in agrammatic aphasia (Grodzinsky, Piñango, Zurif, \& Drai, 1999). A second group adopts the view that syntactic representations are largely intact, but aphasic impairment is a consequence of difficulty processing all elements of these representations due to altered neural dynamics such as slowed activation or inability to maintain or inhibit linguistic information (e.g., Hartsuiker \& Kolk, 1998; Kolk, 1995). This view has motivated interventions that aim to expand the capacity of working memory (e.g., Salis, Hwang, Howard, \& Lallini, 2017), or encourage the speaker to adapt to these constraints through production of simple sentences with reduced syntactic structure or lexical content (Springer, Huber, Schlenck, \& Schlenck, 2000). A third group also adopts the view that syntactic computations are 
largely intact and directs intervention to pre-syntactic levels, with focus on integrating (or mapping) sentence structure with higher-level semantic/thematic information (Marshall, Chiat, \& Pring, 1997; Schwartz, Saffran, Fink, Myers, \& Martind, 1994). These therapies often focus on verb processing and event structure (for example, that a transitive verb such as chase typically requires both a subject and an object, and - in the case of chase - both the subject and object are animate). In general, studies have operated at an early stage of intervention research with small-scale trials employing case series methods.

In this project, we employ insights from a new class of grammatical theories (grouped under the heading of usage-based theories) and, in particular, Construction Grammar (CxG; Croft, 2001; Dąbrowska, 2014; Goldberg, 1995, 2006; Langacker, 1987, 1991). In contrast to traditional generative models of grammar (e.g., Chomsky, 1981, 1995), CxG proposes that speakers' linguistic knowledge is captured in terms of constructions, or linguistic units of varying size and degree of abstractness which are stored in memory. It suggests that all linguistic knowledge consists of constructions, some of which are small and concrete - such as single words, while others are large yet also concrete - such as idioms and formulaic utterances such as you know what I mean or I don't know. Other constructions are semi-fixed and contain open slots into which other constructions can be inserted (e.g., I want a ), while some are fully abstract (e.g., X VERBS Y). Furthermore, CXG differs from standard approaches in not drawing a strict demarcation between grammar and lexicon, and semantics and pragmatics. All constructions are forms paired with meaning and use. Frequency of use determines the degree of specification of the construction: lexical strings that frequently cooccur maybe consolidated into a single representation (Varley \& Whiteside, 2001). High usage frequency reflects high functional value: these relatively concrete constructions are used to manage everyday interactions with other speakers, and express needs and wishes.

CXG provides a coherent account of language learning (Tomasello, 2003) and has motivated earlyphase interventions in children with developmental language disorder (Riches, 2013). The theory is attractive from a neurobiological perspective as it employs, at sentence level, variables such as ageof-acquisition and frequency-of-use that have delivered powerful insights into the nature of lexical processing. Such constructs are easily translatable into models of memory access, storage and retrieval. Importantly for the design of the UTILISE intervention, high frequency concrete constructions may be relatively intact even in severe forms of aphasia and might be used to facilitate access to other linked constructions, thus extending the communicative repertoire of the aphasic speaker (Bruns et al., 2019; Zimmerer et al., 2018). Although not couched in the terminology of CxG, other therapeutic approaches in aphasia have employed formulaic frames. For example, recent 
iterations of constraint-induced aphasia/intensive language-action therapy (Difrancesco, Pulvermüller, \& Mohr, 2012) describe use of interactional formulas. Similarly, formulas are inherent to therapies that focus on practice of conversational scripts for use in specific situations (e.g., Kaye \& Cherney, 2016).

We developed a new computer therapy for sentence comprehension and production impairments in aphasia, with the content motivated by CxG. We opted for a digital intervention to enable close control of the intervention protocol and to explore potential for a future self-administered therapy that would allow for high intensity intervention delivered at times and locations convenient to the user (Varley et al., 2016). We identified constructions of relatively high frequency and interactional value. These constructions are the core of the program content and the therapy involves reinforcing them through priming (Hartsuiker \& Kolk, 1998), facilitating their production through use of errorreduction techniques (Whiteside et al., 2012), and then gradually expanding the communicative options they offer. One component of the intervention employs a word-monitoring task in order to encourage 'real-time' or online language processing (Tyler, Moss, Patterson, \& Hodges, 1997). We tested components of the intervention in two pilot studies, employing case series designs (Bruns et al., in prep.). The pilot studies permitted refinement of the intervention and assessment battery, including outcome measures. They also supported a power calculation to inform the current trial.

We combined the computerised intervention with a form of non-invasive brain stimulation transcranial direct current stimulation (tDCS). There is a growing literature on the use of tDCS in neurorehabilitation (e.g., Darkow, Martin, Würtz, Flöel, \& Meinzer, 2017; Fridriksson et al., 2018). In the case of aphasia, tDCS has been used predominantly in combination with lexical therapies (Flöel, 2014), and of those trials measuring sentence-level outcomes, none have used a CXG framework. Furthermore, the follow-up/maintenance periods in initial studies were typically short (e.g., three weeks in Fridriksson, Richardson, Baker, \& Rorden, 2011). In this study, we randomly allocated half the participants to an active tDCS condition, with the anode placed over the left inferior frontal gyrus (IFG), a region claimed to part of the core language/syntax network (Friederici, 2009; Hagoort \& Indefrey, 2014). The remaining participants were administered sham tDCS (30 seconds stimulation). We implemented a double-blind protocol, masking both the researcher and the participant to stimulation condition. Outcomes of the intervention were assessed at the end of treatment, and after an 8-week no-treatment period to determine the stability of any behavioural change.

Aim: our primary aim is to explore the efficacy of a computer-delivered behavioural therapy, informed by principles of $\mathrm{CxG}$, on aphasic sentence comprehension and production impairments. 
Treatment will be compared to a waiting list/usual care condition. Our secondary aim is to determine the extent to which adjunct tDCS, delivered to the left IFG, enhances treatment outcomes immediately post-intervention and after an 8-week no-treatment maintenance period.

We employed a randomised control trial methodology and the protocol was informed by SPIRIT 2013 (Chan et al., 2013) and TIDieR guidelines (Hoffmann et al., 2014). Trial outcome reports will conform to CONSORT 2010 (Schulz, Altman, \& Moher, 2010) principles.

\section{Research questions}

(1) Does the computerised behavioural therapy improve connected speech production and spoken sentence comprehension in comparison to waitlist/usual care control;

(2) Does anodal tDCS delivered to the left IFG enhance treatment outcomes immediately after treatment and at an 8-week maintenance assessment;

(3) What is the relationship between treatment outcome and demographic, behavioural variables, lesion size and location;

(4) What is the impact of the intervention on perceptions of quality-of-life, and on an untreated control language behaviour.

Ethical approval for the research was granted by the UCL Research Ethics Committee on 12/06/2019 $(8123 / 001)$.

\section{Methods}

UTILISE is a single centre randomised control exploratory trial, comparing the outcomes of a behavioural therapy for aphasic sentence processing impairments with a deferred trial entry/usual care condition. Further, it will examine the effectiveness of double-blinded active versus sham tDCS in combination with the behavioural intervention. Stability of behaviour before intervention will be established from two baseline assessments. Outcomes will be measured immediately postintervention and after an 8-week no treatment maintenance period. Figure 1 presents the study design. 


\begin{tabular}{|c|c|c|c|c|c|c|c|}
\hline & Enrolment & $\begin{array}{c}\text { Baseline } \\
1\end{array}$ & Allocation & $\begin{array}{l}\text { Baseline } \\
\quad 2^{*}\end{array}$ & Therapy & $\begin{array}{c}\text { Outcome } \\
1\end{array}$ & Maintenance \\
\hline $\begin{array}{l}\text { Time point } \\
\text { (weeks) }\end{array}$ & -1 & 0 & 0 & 4 & $5-8$ & 9 & 17 \\
\hline \multicolumn{8}{|l|}{ ENROLMENT } \\
\hline Eligibility screen & $x$ & & & & & & \\
\hline Consent form & & $x$ & & & & & \\
\hline $\begin{array}{l}\text { Inclusion / } \\
\text { exclusion form \& } \\
\text { tDCS/MRI safety } \\
\text { screen }\end{array}$ & & $\mathrm{x}$ & & & & & \\
\hline $\begin{array}{l}\text { Demographics } \\
\text { questionnaire }\end{array}$ & & $x$ & & & & & \\
\hline Randomisation & & & $x$ & & & & \\
\hline \multicolumn{8}{|l|}{ INTERVENTION } \\
\hline $\begin{array}{l}\text { 4-week therapy } \\
\text { program }\end{array}$ & & & & & $x$ & & \\
\hline \multicolumn{8}{|l|}{ ASSESSMENTS } \\
\hline \multicolumn{8}{|c|}{ CONTROL MEASURE } \\
\hline $\begin{array}{l}\text { Synonym } \\
\text { matching task }{ }^{1}\end{array}$ & & $\mathrm{x}$ & & $\mathrm{x}$ & & $\mathrm{x}$ & $x$ \\
\hline \multicolumn{8}{|c|}{ OUTCOME MEASURES } \\
\hline TROG- $2^{2}$ & & $x$ & & $\mathrm{x}$ & & $\mathrm{x}$ & $x$ \\
\hline $\begin{array}{l}\text { Personal } \\
\text { narrative }^{3}\end{array}$ & & $x$ & & $\mathrm{x}$ & & $\mathrm{x}$ & $x$ \\
\hline $\begin{array}{l}\text { Picture-based } \\
\text { narrative }^{4}\end{array}$ & & $x$ & & $\mathrm{x}$ & & $\mathrm{x}$ & $x$ \\
\hline $\begin{array}{l}\text { Story completion } \\
\text { test }^{5}\end{array}$ & & $x$ & & & & $x$ & \\
\hline SAQOL-396 & & & & $x$ & & & $x$ \\
\hline \multicolumn{8}{|c|}{ PROFILING MEASURES } \\
\hline $\begin{array}{l}\text { Edinburgh } \\
\text { Handedness } \\
\text { Inventory (short } \\
\text { form) }{ }^{7}\end{array}$ & & $\mathrm{x}$ & & & & & \\
\hline PALPA- $13^{8}$ & & $x$ & & & & & \\
\hline The Brixton Test ${ }^{9}$ & & & & $x$ & & & \\
\hline $\begin{array}{l}\text { WASI-II Matrix } \\
\text { Reasoning }{ }^{10}\end{array}$ & & & & $x$ & & & \\
\hline
\end{tabular}




\begin{tabular}{|l|l|l|l|l|l|l|l|}
\hline $\begin{array}{l}\text { CAT spoken word } \\
\text { comprehension }\end{array}$ & & & & $\mathrm{X}$ & & & \\
\hline $\begin{array}{l}\text { Boston Naming } \\
\text { Test }\end{array}$ & & & & $\mathrm{X}$ & & & \\
\hline OTHER MEASURES & & & & & $\mathrm{X}$ & \\
\hline $\begin{array}{l}\text { Feedback } \\
\text { questionnaire }\end{array}$ & & & & & & & \\
\hline
\end{tabular}

Figure 1: Study design for UTILISE trial

* 4-week interval between Baseline 1 and 2 for immediate group versus 8-week interval for deferred group.

${ }^{1}$ Shortened form of Written Synonym Matching Task, taken from the Action for Dysphasic Adults Auditory Comprehension Battery (A.D.A.), Franklin et al., 1992; ${ }^{2}$ Test for Reception of Grammar Version 2 (TROG-2), Bishop, 2003; ${ }^{3}$ Spontaneous speech: questions to elicit personal narratives; ${ }^{4}$ Fletcher \& Birt, 1983; ${ }^{5}$ adapted from Goodglass et al., 1972; ${ }^{6}$ The Stroke and Aphasia Quality of Life Scale (SAQOL-39), Hilari et al., 2003; ${ }^{7}$ aphasia-friendly version adapted from Veale, $2014 ;{ }^{8}$ Auditory Digit Repetition Span / digit pointing task: Subtest 13 from the Psycholinguistic Assessments of Language Processing in Aphasia (PALPA): Kay, Coltheart, \& Lesser, $1992 ;{ }^{9}$ Burgess \& Shallice, 1997; ${ }^{10}$ WASI-II Matrix Reasoning subtest: Wechsler Abbreviated Scale of Intelligence - Second Edition: Wechsler, 2011; ${ }^{11}$ Comprehensive Aphasia Test (CAT), Swinburn, Porter, \& Howard, 2004; ${ }^{12}$ Kaplan, Goodglass, \& Weintraub, 2001 
Setting: this efficacy study will take place in University College London.

Population: 66 adults with post-stroke aphasia and impairments in spoken sentence comprehension and/or production will be recruited to the study. Inclusion and exclusion criteria for the study are provided in Table 1.

\begin{tabular}{|c|c|}
\hline Inclusion criteria & Exclusion criteria \\
\hline $\begin{array}{l}\text { - Premorbid competence in English } \\
\text { - Premorbidly right handed } \\
\text { - } \text { Single left hemisphere stroke } \\
\text { - At least six-months post-stroke onset } \\
\text { - Presence of moderate-to-severe aphasia, } \\
\text { characterised by spoken sentence } \\
\text { comprehension impairment (scoring < } 16 \\
\text { blocks correct on TROG-2) and/or } \\
\text { spoken sentence production difficulties } \\
\text { (incomplete and/or simple sentences) at } \\
\text { baseline assessment } \\
\text { - Capacity to give informed consent } \\
\text { - Sufficient auditory and visual capacity to } \\
\text { interact with the behavioural therapy }\end{array}$ & 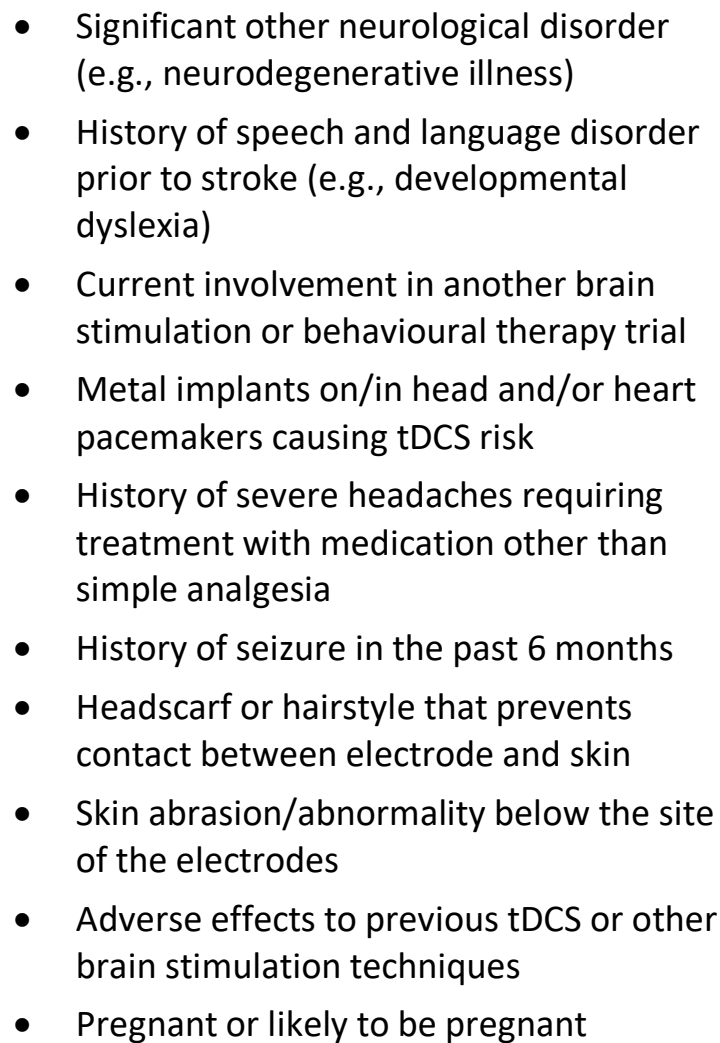 \\
\hline
\end{tabular}

Table 1: Inclusion and exclusion criteria employed in trial recruitment

Identification and recruitment: The first phase of recruitment will identify volunteers from a university research register who fit to the study inclusion/exclusion criteria. Potential recruits will be sent an information sheet and directed to online recruitment materials (https://www.youtube.com/watch?v=hwLZLQFmrsY and https://www.cognitionandgrammar.net/utilise)

Other potential recruits will be approached via community and voluntary sector groups. Potential recruits are given two weeks to decide if they wish to join the trial before informed consent is taken. Information sheets, consent forms and other recruitment materials are designed to be aphasia friendly in order to circumvent difficulties in understanding spoken and/or written information. We will record invitations, acceptance rates and reasons for non-participation (if any are offered) and 
report these data in a CONSORT figure. Volunteers will be screened for suitability and match to the study inclusion/exclusion criteria. A second phase of recruitment from post-acute NHS speech and language therapy services will subsequently be launched, employing these same procedures once IRAS approval is gained.

Randomisation: after first baseline assessment, participants who fit recruitment criteria are randomised to either immediate or deferred trial entry, and active vs. sham tDCS conditions. Randomisation is performed via a computer-generated code from an external randomisation service (www.sealedenvelope.com). Participants are stratified by sex and randomised on a 2:2:1:1 basis to one of four conditions: immediate treatment + active tDCS; immediate treatment + sham tDCS; deferred treatment + active tDCS; deferred treatment + sham tDCS. Block randomisation is employed, with the clinical researchers blind to block size. After randomisation, allocation is communicated to the researcher via a sequential number system.

Blinding: Allocation to treatment condition is assigned by a member of the research team who is blind to case. Researchers and participants cannot be blinded to immediate versus deferred trial entry status, thus the design is an open-label trial for the behavioural component. The tDCS component of the trial is double-blinded as neither the participant nor researcher administering stimulation is aware of the active/sham allocation. Transcribers of audio-recordings of narrative speech samples (the primary outcome measure) and responses on the story completion test (a secondary outcome measure) will be masked to both phase of the sample and tDCS condition. Each sample will be allocated a code to mask its status as a baseline or post-intervention measure. Any comments by the participant that reveals the timing of the assessment will be edited from the audio file. Transcribers will be junior researchers who will not be present when the sample is recorded. For the narrative samples, recordings will be transcribed to orthography and tagged with the conventions necessary for processing by FLAT software (Zimmerer et al., 2016). Fidelity of transcriptions and tagging will be determined via sample inter-reliability evaluation. Changes in spoken sentence comprehension will be assessed with the TROG-2 (Bishop, 2003). The assessor will be masked to tDCS allocation, but not to phase. Evaluations will be video-recorded, allowing fidelity checks on the administration of the test and the accuracy of scoring, and conducted by a researcher masked to condition and phase.

Masking of active/sham tDCS allocation will be maintained until primary data analysis is complete. The only circumstances in which a participant's allocation would be unblinded would be if they experienced a seizure or other adverse event during or soon after the trial. 
Sample size: Based on a pilot case series with six participants, sample size was calculated with formula $n=2+C(s / d)$ (Note that for $\alpha=0.05$ and $1-\beta=0.9, C=10.51$ ), on the basis of an estimation of the population standard deviation (s) and the expected difference (d). Sample size estimation for mixed-factor ANOVA with a factorial design (session Baseline-2/Outcome-1) within subject $\times$ group active/sham between subject) was also performed with a specialised software (G*Power; Erdfelder, Faul, Buchner, \& Lang, 2009). Both approaches suggested a sample size close to 22 participants per group.

\subsection{Outcome measures}

On entry to the trial, we collect background data (age, education, employment, language history, medical history relevant to the study (e.g., date of stroke)). Participants' language and cognitive abilities will be profiled on standardised tests. Table 2 lists these tests and the capacities they measure.

\begin{tabular}{|l|l|}
\hline \multicolumn{1}{|c|}{ Test } & \multicolumn{1}{c|}{ Capacity } \\
\hline $\begin{array}{l}\text { Edinburgh handedness inventory - short form } \\
\text { (Veale, 2014) }\end{array}$ & Premorbid laterality \\
\hline $\begin{array}{l}\text { Wechsler Abbreviated Scale of Intelligence } \\
\text { (WASI-II) Matrix Reasoning } \\
\text { (Wechsler, 2011) }\end{array}$ & Non-verbal IQ \\
\hline $\begin{array}{l}\text { Brixton Spatial Anticipation Test } \\
\text { (Burgess \& Shallice, 1997) }\end{array}$ & Non-verbal executive function \\
\hline $\begin{array}{l}\text { The Comprehensive Aphasia Test: } \\
\text { comprehension of spoken words } \\
\text { (Swinburn, Porter, \& Howard, 2004) }\end{array}$ & Auditory lexical comprehension \\
\hline $\begin{array}{l}\text { Boston Naming Test } \\
\text { (Kaplan, Goodglass, \& Weintraub, 2001) }\end{array}$ & Spoken lexical retrieval \\
\hline $\begin{array}{l}\text { Digit span (spoken) } \\
\text { (Kay, Coltheart, \& Lesser, 1992) }\end{array}$ & Phonological working memory \\
\hline
\end{tabular}

Table 2: Behavioural profiling battery

The primary outcome measure for the study is connectivity of speech (ratio of three-word combinations (trigrams) to total number of words) in narrative tasks (personal narrative, e.g., describing a recent holiday, and retelling picture cartoon series (Fletcher \& Birt, 1983)). Speech will be audio-recorded, orthographically transcribed and tagged for analysis by automated analysis software (FLAT, Zimmerer et al., 2016). 
The secondary outcome measures are spoken sentence comprehension, measured with TROG-2 (Bishop, 2003), an adapted version of the story completion test (Goodglass et al., 1972) that aims to elicit the trained sentence constructions and roughly matched untreated constructions, and the SAQOL-39 (Hilari et al., 2003) which measures perceptions of quality-of-life. Performance on a shortened version of the A.D.A Written Synonym Judgement Test (Franklin et al., 1992) will be assessed to determine change on an untreated language behaviour. Assessors are trained to use standardised instructions for administration of all measures. Participants will complete assessments across two baseline sessions to determine stability of scores on outcome measures prior to intervention. The story completion test is a lengthy assessment and will be administered only at the first baseline assessment and the first outcome evaluation. There will be approximately four-weeks between baselines for the immediate trial entry group, and approximately eight-weeks for the deferred trial entry group. Assessments and intervention are delivered by research assistants employed on the trial. Measures are repeated immediately at the end of treatment (primary endpoint) and after an 8-week maintenance period (secondary endpoint).

\subsection{Intervention}

Computer-delivered behavioural therapy will be delivered in 12 face-to-face sessions in a university setting over a four-week period. It will be administered in individual sessions by trained research staff (qualified to post-graduate level in relevant disciplines). Each session lasts for approximately 60 minutes (20 minutes tDCS set-up; 40 minutes therapy) and are scheduled on alternate days across the week (Monday, Wednesday, Friday). UTILISE therapy consists of three tasks: two involve listening to sentences and one spoken production. In task one, participants listen to two sentences. Sentences are always different at a surface perceptual level (one produced by a female speaker and the other by a male speaker). Participants judge whether the speakers say the same or different phrases/sentences. 'Different' sentences are changed by one word that is located in subject, verb, object or adjunct position and usually the change is on a content rather than a function word. Stimuli include the high frequency constructions that appear in the later production task (task 3 ) in order to prime these structures. Across all trials, proportionally there are more changes of verbs ( $41 \%$ of trials are verb manipulations), reflecting the central role of the verb in governing the structure of the sentence. The software includes an automatic ladder algorithm that adjusts difficulty level in response to the participant's performance. Difficulty can be increased through longer sentence length and retention interval between sentences (2000 to $4000 \mathrm{msec}$ ). After a block of 30 trials, participants receive feedback on accuracy. 
The second task also involves listening to spoken sentences, including the target constructions that appear in task 3. We will employ a word-monitoring task to encourage rapid processing of sentence strings. Participants are presented with a target word and have to press a button as soon as they hear that word in the sentence string. Proportionally, there are again more verb targets in the stimuli. Participants receive feedback on accuracy and speed of response.

The final task stimulates spoken production of target constructions. It employs error reduction principles (Whiteside et al., 2012). First, participants listen to an audio recording of the target sentence. They are then encouraged to imagine saying it in unison with further audio presentations. Finally, they are encouraged to say the string aloud, with the option to record and subsequently listen back to their performance. After stimulation with the high frequency variant of the string (e.g., I had it), alternative lexical items are placed in either the subject or object slots (e.g., I had breakfast, I had pain, She had it, He had it). Subsequently, new lexical options are placed in both of these slots (She had breakfast, He had pain), or an adjunct is added to the phrase (e.g., I had it today). The overarching aim is to stimulate greater productive/creative capacity. Screenshots of tasks and an example of a construction and its expansion are provided on the project website https://www.cognitionandgrammar.net/utilise. Participants take home their best recordings (up to 25) on a private memory stick.

The behavioural therapy will be combined with active or sham tDCS delivered by a DC-Stimulator (neuroConn, Ilmenau, Germany). In initial allocation, the participant is given a numeric code that drives either active or sham tDCS. Both the participant and the researcher are masked to the tDCS stimulation condition. Prior to intervention, participants complete a standard safety screening for tDCS. Subject to satisfactory screening, half of the participants will receive active stimulation and half sham stimulation. All participants have the anodal electrode placed over the left inferior frontal region (close to $\mathrm{F} 5$ of the extended International 10-20 system for EEG electrode placement) and the cathode on the right wrist (electrode size $5 \times 7 \mathrm{~cm}$ ). In the active condition, stimulation parameters are $20 \mathrm{~min}$ at $1.5 \mathrm{~mA}$ with 15 seconds ramp-up and -down. In the sham condition, the current is ramped up to $1.5 \mathrm{~mA}$ and ramped down over 30 seconds to ensure the typical initial tingling sensation. Stimulation will be delivered at the start of each therapy session, conjointly with the behavioural intervention.

At the end of the intervention, participants are screened for MR safety and will have a structural MRI brain scan (1.5T Siemens Avanto scanner) to determine the integrity of tissue in the left inferior frontal region, and the location and extent of lesion. 
Outcomes of treatment will be compared to usual care. Usual care will consist of no change to the participant's rehabilitation regime. We will collect information on services received. During the treatment phase of the trial, participants will be requested to undertake no other impairmentorientated language therapies.

\subsection{Treatment fidelity}

To maintain treatment fidelity, therapy manuals have been produced for both the computer and tDCS interventions. The language tasks are fixed in content, structure and progression. However, the researcher has a small amount of flexibility in allocating time to each task, dependent upon the participant's success level. The software records the participant's interactions with the program and thus match to the recommended protocol can be determined. Any violations of the protocol are noted in the participant's record.

\subsection{Acceptability of intervention}

Participants complete an acceptability questionnaire and have the opportunity to make further comments on the content and processes of the study after completion of therapy. Participants will be offered the opportunity to join a focus group to seek additional feedback on the intervention and study processes.

\subsection{Data management}

Participants are allocated an anonymous code at study entry and all data are labelled with this code. Using opt-in consent, participants can decide if their anonymised data can be shared with other researchers, for example, in support of meta-analyses of treatment outcomes. Personal narratives can contain identifying information and therefore proper names (people and places) are removed from the sample during transcription. The research is GDPR compliant. There is no data monitoring committee due to funding limits and the early phase status of the trial. Data will be analysed with an intention-to-treat criterion. There will be no imputation of missing values.

The outcome data are quantitative. These include the ratio of three-word combinations (trigrams) to total number of words in narratives. Scores on TROG-2, the story completion test, SAQOL-39 and written synonym judgement are also analysed. Data accuracy against paper records will be checked once final data entry is complete. We will conduct analyses linked to each research question. Data will be assessed for fit to parametric criteria and planned analyses include mixed analysis of variance (ANOVA) with a within-participant factor of Time and a between-participant factor of Group. 
Significant main effects and interactions will be explored with post hoc $t$ tests, with Bonferroni corrections for multiple comparisons. We will examine the baseline profile characteristics of the groups (e.g., age, education, time post-stroke) with independent sample $t$-tests, adjusting for significant differences through co-variates where required. We will conduct exploratory analyses regarding correlates of positive treatment outcome (calculated from difference scores between baseline 2 and outcome 1) through regression analysis including demographic characteristics, baseline profiling and lesion volume and location.

Qualitative data will be collected on participants' feedback on the intervention. We will record any adverse events from tDCS or MRI in order to inform development of future safety criteria. Adverse events will be reported to the relevant ethics committee and the protocol re-evaluated and, where deemed necessary, revised. Any revisions will be posted to the ISRCTN registry record. We will also record reasons (if offered) for dropping out of the trial.

\subsection{Assessment and management of risk}

The behavioural intervention involves minimal risk for participants. tDCS contraindications include metal in the head, pacemakers, history of seizure within the last six months, and severe and recurrent headaches (Table 1). There is also risk of irritation of skin under electrodes. These risks are managed first by a screening assessment of suitability for tDCS. Presence and severity of headache is recorded at the start and end of a session. Skin is assessed for signs of irritation when electrodes are placed, removed and again at the start of the next session. If risks are identified, researchers refer to the investigator team and, if necessary, further expert or clinical opinion is sought. Where there is judged to be a risk to a participant, no further tDCS will be administered and the participant can opt to continue with behaviour-only intervention or drop out of the study. Participants who drop out of the study will be invited to attend outcome assessments

With regard to MRI, contraindications include implanted electronic medical devices, the presence of metal or metal-containing foreign bodies in the body as a result of injury or medical procedures (with some exceptions for non-recent fixed metallic dental or bone implants), pregnancy, claustrophobia. These risks are managed first by completion of a screening questionnaire at trial entry and a follow-up interview conducted by a qualified member of the investigator team. Immediately prior to the imaging session, the safety screening questionnaire is repeated, and participants remove metal-containing clothing and objects prior to scanning, and final metal-free status is checked with a hand-held metal detector prior to entering the scanner's magnetic field. In 
the event of contraindications to MRI, the participant will not be scanned. However, their behavioural data will be included in the outcome analysis of the trial.

\section{Discussion}

This study aims to innovate therapy for sentence processing impairments in aphasia. It applies a theory of grammatical behaviour - CxG - that has yet to be fully exploited in the language therapy field. CxG may have particular value in motivating therapy: its usage-based principles lead to focus on frequent constructions with high functional value in everyday communication. Furthermore, its emphasis on storage of constructions in memory, with relatively simple principles by which to fill empty slots in more abstract frames, contrasts with more traditional grammatical approaches. As a result, CXG has strong neurobiological plausibility and provides a coherent foundation on which to develop grammatical therapies.

The behavioural intervention employed in this study is computerised and we will explore the acceptability of the program to participants and its potential for refinement for an autonomouslydelivered therapy. Previous investigations and meta-analyses have indicated the importance of high dose level in therapy outcome. However, high dose is difficult to achieve in therapist-delivered treatment due to cost implications, but also in acceptability to patients and their families, particularly in the early phase of stroke recovery (Bhogal, Teasell, \& Speechley, 2003). Software interventions that can be self-administered, with therapist support, represent a means to deliver high-dose therapy at times and locations convenient to the user and, at the same time, maintaining fidelity of the intervention (Varley, 2011).

The study will be the first to employ the FLAT analysis software in a larger scale intervention trial. The FLAT profiles connected speech on multiple dimensions. In addition to the connectivity analysis that we use as a primary outcome measure, it generates a range of variables that provide insight into language production. For example, there is automatic analysis of the frequency of content and function words, as well as the frequency of bigram and trigram combinations (Bruns et al., in prep., Zimmerer, Wibrow, \& Varley, 2016). Type-token ratios permit quantification of the lexical diversity within a sample. The analysis is automated and so is fast and blind, making it ideal tool for use in intervention research. Subsequent data-mining studies will allow us to explore the optimal variables, or combination of variables, for use in characterising language change in aphasia. We will also be able to explore variable-lesion associations through linkage of language profiles to lesion analysis. 
A number of studies have examined the enhancement of therapy outcomes when behavioural intervention is combined with tDCS. Most of the published studies have explored this issue in relation to lexical therapy. The current project will determine the value of tDCS in supporting sentence-level therapy. Furthermore, most studies that have employed tDCS have relatively short follow-up periods (Stahl et al., 2019). One possibility is that, due to action at the neuronal level, tDCS stabilises behavioural gain (Javadi, Brunec, Walsh, Penny \& Spiers, 2014). The longer eight-week maintenance period used by this study will allow evaluation of this claim.

The UTILISE project will establish a database that will be available for future meta-analyses of aphasia treatment outcomes. We and other researchers will be able to explore characteristics of sub-groups of participants who display different responses to the interventions. Baselines profiles of severity of aphasic impairment, chronicity, and biographical characteristics such as age, sex and education, will permit development of prognostic indicators for therapy. We will also understand more of the impact of lesion location and size on grammatical behaviour and response to therapy. Subsequently this will support personalised medicine initiatives that allow allocation of individuals to the most effective management regimes.

\section{Possible limitations}

The UTILISE trial, with a target recruitment of 66 participants divided into three participant groups, is larger than most previous investigations of therapy for sentence processing impairments in aphasia. However, participant numbers remain relatively small, limiting sub-group analysis. The first phase of recruitment draws volunteers from a university research register, which might bias selection towards chronic phase participants who are highly motivated to contribute to research. If the initial trial outcomes are promising, future investigations would be warranted that examine effectiveness in larger samples, with participants recruited in acute/sub-acute recovery phase and from typical clinical services.

With regard to outcome measures, our choice of connectivity from the FLAT as the primary measure is based upon small-scale pilot studies. One possibility is that other variables or combinations of variables are more suitable for profiling outcomes. We will explore this issue in post-hoc analysis in order to make recommendations for future research. All the outcome measures will be administered by a researcher who is not blind to phase of the assessment. Although we have incorporated into the study design procedures for data fidelity checks, ideally outcomes would be assessed by a researcher blind to phase. Funding limitations preclude this option. 
The dose level employed in this study (3-hours per week for 4-weeks) is below that reported to be effective in aphasia rehabilitation. If the outcomes of this Phase-II trial are promising, future studies could increase the length and intensity of the treatment period. Alternatively, the intervention software could be designed to support home/self-administration. We combine computerised behavioural intervention with tDCS. Candidates for tDCS have to pass a safety screen in order to exclude individuals for whom brain stimulation is contraindicated. Some of the contraindications are relatively common in patients following stroke (e.g., recent seizure, metal implants such as aneurysm coils, severe/recurrent headache). We will record numbers of volunteers who are excluded from the trial on the basis of tDCS safety criteria and monitor the impact on overall recruitment. If faced with large numbers of exclusions, we will consider running a parallel behaviouronly study, where participants are randomised to immediate or deferred trial entry and receive only the computerised behavioural therapy. The outcomes of this study would be reported separately from the main trial.

\section{Dissemination plan}

The outcomes of the research will be reported in clinical-academic journals and at conferences. We will also engage with service users at special events and develop aphasia-friendly ways to report the methods and results of the study. Anonymised primary data will be available to other researchers, subject to participant consent to data sharing. A detailed therapy manual will be available from the research team on reasonable request. 
Trial status: ISRCTN14466044. Recruitment began on $18^{\text {th }}$ September 2019 and is due to end 1st December 2021.

Abbreviations: CxG - Construction Grammar; FLAT - Frequency in Language Analysis Tool; tDCS transcranial direct current stimulation.

Acknowledgements: We thank Michael Coleman for software programming; Michael Dean for assistance in participant recruitment; Richard Jardine for his time in making audio recordings; Vitor Zimmerer for access and support in use of the FLAT software.

Availability of data and materials: Project information sheet, consent form and sample therapy materials available at https://www.cognitionandgrammar.net/utilise. Anonymised data (subject to participant consent) will be made available at the end of the trial in an open science repository.

Author contributions: RV and CB wrote the manuscript; ED, AJH and JW contributed to the revision of the manuscript. All authors read and agreed the final version of the manuscript.

Ethics approval: All participants will give informed consent to take part in the study.

Competing interests: None 


\section{References}

Berthier, M. L. (2005). Poststroke aphasia: Epidemiology, pathophysiology and treatment. Drugs and Aging, 22(2), 163-182. https://doi.org/10.2165/00002512-200522020-00006

Bhogal, S. K., Teasell, R., \& Speechley, M. (2003). Intensity of aphasia therapy, impact on recovery. Stroke, 34(4), 987-992. https://doi.org/10.1161/01.STR.0000062343.64383.D0

Bishop, D. V. M. (2003). Test for Reception of Grammar, Version 2 (TROG-2). London: Pearson.

Brady, M., Kelly, H., Godwin, J., Enderby, P., \& Campbell, P. (2016). Speech and language therapy for aphasia following stroke. Cochrane Database of Systematic Reviews, (6). https://doi.org/10.1002/14651858.CD000425.pub4.www.cochranelibrary.com

Bruns, C., Varley, R., Zimmerer, V. C., Carragher, M., Brekelmans, G., \& Beeke, S. (2019). “I don't know": a usage-based approach to familiar collocations in non-fluent aphasia. Aphasiology, 2(33), 140-162. https://doi.org/10.1080/02687038.2018.1535692

Bruns, C., Beeke, S., Zimmerer, V. C., Coleman, M., Bruce, C., \& Varley, R. (in prep). Training flexibility in fixed expressions in non-fluent aphasia - a case series report.

Burgess, P. W., \& Shallice, T. (1997). The Hayling and Brixton tests: Two tests of dysexecutive syndrome. Bury St Edmunds: Thames Valley Test Company.

Chan, A.-W., Tetzlaff, J. M., Gøtzsche, P. C., Altman, D. G., Mann, H., Berlin, J. A., ... Krleža-Jeric, K. (2013). SPIRIT 2013 explanation and elaboration: guidance for protocols of clinical trials. BMJ (Clinical Research Ed.), 346, 1-42. https://doi.org/10.1136/bmj.e7586

Chomsky, N. (1981). Lectures on Government and Binding: The Pisa Lectures. Berlin: Walter de Gruyter.

Chomsky, N. (1995). The Minimalist Program. Cambridge, MA: MIT Press.

Croft, W. (2001). Radical construction grammar: Syntactic theory in typological perspective. Oxford: Oxford University Press. https://doi.org/10.1093/acprof:oso/9780198299554.001.0001

Dąbrowska, E. (2014). Recycling utterances: A speaker's guide to sentence processing. Cognitive Linguistics, 25, 617-653. https://doi.org/10.1515/cog-2014-0057

Darkow, R., Martin, A., Würtz, A., Flöel, A., \& Meinzer, M. (2017). Transcranial direct current stimulation effects on neural processing in post-stroke aphasia. Human Brain Mapping, 38(3), 1518-1531. https://doi.org/10.1002/hbm.23469

Difrancesco, S., Pulvermüller, F., \& Mohr, B. (2012). Intensive language-action therapy (ILAT): The 
methods. Aphasiology, 26(11), 1317-1351. https://doi.org/10.1080/02687038.2012.705815

Edmonds, L. A. (2016). A review of Verb Network Strengthening Treatment: Theory, methods, results, and clinical implications. Topics in Language Disorders, 36(2), 123-135.

https://doi.org/10.1097/TLD.0000000000000088

Erdfelder, E., Faul, F., Buchner, A., \& Lang, A.-G. (2009). Statistical power analyses using G*Power 3.1: Tests for correlation and regression analyses. Behavior Research Methods, 41(4), 11491160. https://doi.org/10.3758/BRM.41.4.1149

Fletcher, M., \& Birt, D. (1983). Storylines: Picture sequences for language practice. London: Longman.

Flöel, A. (2014). tDCS-enhanced motor and cognitive function in neurological diseases. Neurolmage, 85, 934-947. https://doi.org/10.1016/j.neuroimage.2013.05.098

Franklin, S., Turner, J., \& Ellis, A. (1992). The A.D.A. Auditory Comprehension Battery. York, UK: University of York.

Fridriksson, J., Richardson, J. D., Baker, J. M., \& Rorden, C. (2011). Transcranial direct current stimulation improves naming reaction time in fluent aphasia: A double-blind, sham-controlled study. Stroke, 42(3), 819-821. https://doi.org/10.1161/STROKEAHA.110.600288

Fridriksson, J., Rorden, C., Elm, J., Sen, S., George, M. S., \& Bonilha, L. (2018). Transcranial Direct Current Stimulation vs Sham Stimulation to Treat Aphasia after Stroke: A Randomized Clinical Trial. JAMA Neurology, 75(12), 1470-1476. https://doi.org/10.1001/jamaneurol.2018.2287

Friederici, A. D. (2009). Pathways to language: fiber tracts in the human brain. Trends in Cognitive Sciences, 13(4), 175-181. https://doi.org/10.1016/j.tics.2009.01.001

Goldberg, A. E. (1995). A Construction Grammar Approach to Argument Structure. Chicago, IL: The University of Chicago Press.

Goldberg, A. E. (2006). Constructions at Work: The Nature of Generalization in Language. New York: Oxford University Press. https://doi.org/10.1093/acprof:oso/9780199268511.001.0001

Goodglass, H., Gleason, J. B., Ackerman Bernholtz, N., \& Hyde, M. R. (1972). Some Linguistic Structures in the Speech of a Broca's Aphasic. Cortex, 8(2), 191-212. https://doi.org/10.1016/S0010-9452(72)80018-2

Grodzinsky, Y., Piñango, M. M., Zurif, E., \& Drai, D. (1999). The critical role of group studies in neuropsychology: Comprehension regularities in Broca's aphasia. Brain and Language, 67(2), 134-147. https://doi.org/10.1006/brln.1999.2050 
Hagoort, P., \& Indefrey, P. (2014). The Neurobiology of Language Beyond Single Words. Annual Review of Neuroscience, 37(1), 347-362. https://doi.org/10.1146/annurev-neuro-071013013847

Hartsuiker, R. J., \& Kolk, H. H. J. (1998). Syntactic Facilitation in Agrammatic Sentence Production. Brain and Language, 62(2), 221-254. https://doi.org/10.1006/brln.1997.1905

Hilari, K., Byng, S., Lamping, D. L., \& Smith, S. C. (2003). Stroke and aphasia quality of life scale-39 (SAQOL-39): Evaluation of acceptability, reliability, and validity. Stroke, 34(8), 1944-1950. https://doi.org/10.1161/01.STR.0000081987.46660.ED

Hoffmann, T. C., Glasziou, P. P., Boutron, I., Milne, R., Perera, R., Moher, D., ... Michie, S. (2014). Better reporting of interventions: Template for intervention description and replication (TIDieR) checklist and guide. BMJ (Online), 348(March), 1-12.

https://doi.org/10.1136/bmj.g1687

Javadi, A. H., Brunec, I. K., Walsh, V., Penny, W. D., \& Spiers, H. J. (2014). Transcranial electrical brain stimulation modulates neuronal tuning curves in perception of numerosity and duration. Neurolmage, 102(P2), 451-457. https://doi.org/10.1016/j.neuroimage.2014.08.016

Kaplan, E., Goodglass, H., \& Weintraub, S. (2001). Boston Naming Test. 2nd Ed. Philadelphia, PA: Lippincott Williams \& Wilkins.

Kay, J., Coltheart, M., \& Lesser, R. (1992). Psycholinguistic Assessments of Language Processing in Aphasia. Hove, UK: Lawrence Erlbaum Associates.

Kaye, R. C., \& Cherney, L. R. (2016). Script Templates: A Practical Approach to Script Training in Aphasia. Topics in Language Disorders, 36(2), 136-153.

https://doi.org/10.1097/TLD.0000000000000086

Kolk, H. H. J. (1995). A Time-Based Approach to Agrammatic Production. Brain and Language, 50(3), 282-303. https://doi.org/10.1006/brln.1995.1049

Langacker, R. W. (1987). Foundations of cognitive grammar. Volume 1. Theoretical prerequisites. Stanford, CA: Stanford University Press.

Langacker, R. W. (1991). Foundations of cognitive grammar. Volume II. Descriptive Application. Stanford, CA: Stanford University Press.

Marshall, J., Chiat, S., \& Pring, T. (1997). An impairment in processing verbs' thematic roles: A therapy study. Aphasiology, 11(9), 855-876. https://doi.org/10.1080/02687039708250461

Palmer, R., Dimairo, M., Cooper, C., Enderby, P., Brady, M., Bowen, A., ... Chater, T. (2019). Selfmanaged, computerised speech and language therapy for patients with chronic aphasia post- 
stroke compared with usual care or attention control (Big CACTUS): a multicentre, singleblinded, randomised controlled trial. The Lancet Neurology, 18(9), 821-833.

https://doi.org/10.1016/S1474-4422(19)30192-9

Riches, N. G. (2013). Treating the passive in children with specific language impairment: A usagebased approach. Child Language Teaching and Therapy, 29(2), 155-169. https://doi.org/10.1177/0265659012466667

Ross, K. B., \& Wertz, R. T. (2003). Quality of life with and without aphasia. Aphasiology, 17(4), 355364. https://doi.org/10.1080/02687030244000716

Salis, C., Hwang, F., Howard, D., \& Lallini, N. (2017). Short-Term and Working Memory Treatments for Improving Sentence Comprehension in Aphasia: A Review and a Replication Study. Seminars in Speech and Language, 38(1), 29-39. https://doi.org/10.1055/s-0036-1597262

Schulz, K. F., Altman, D. G., \& Moher, D. (2010). CONSORT 2010 Statement: Updated guidelines for reporting parallel group randomised trials. BMJ (Online), 340(7748), 698-702. https://doi.org/10.1136/bmj.c332

Schwartz, M. F., Saffran, E. M., Fink, R. B., Myers, J. L., \& Martind, N. (1994). Mapping therapy : A treatment programme for agrammatism. Aphasiology, 8(1), 19-54. https://doi.org/10.1080/02687039408248639

Springer, L., Huber, W., Schlenck, K.-J., \& Schlenck, C. (2000). Agrammatism: Deficit or compensation? Consequences for aphasia therapy. Neuropsychological Rehabilitation: An International Journal, 10(3), 279-309. https://doi.org/10.1080/096020100389165

Stahl, B., Darkow, R., von Podewils, V., Meinzer, M., Grittner, U., Reinhold, T., ... Flöel, A. (2019). Transcranial direct current stimulation to enhance training effectiveness in chronic post-stroke aphasia: A randomized controlled trial protocol. Frontiers in Neurology, 10(OCT), 1-8. https://doi.org/10.3389/fneur.2019.01089

Stroke Association (2018). Retrieved from: https://www.stroke.org.uk/sites/default/files/state_of_the_nation_2018.pdf

Swinburn, K., Porter, G., \& Howard, D. (2004). The Comprehensive Aphasia Test. Hove, UK: Psychology Press.

Thompson, C. K., Choy, J. J., Holland, A., \& Cole, R. (2010). Sentactics ${ }^{\circledR}$ : Computer-Automated Treatment of Underlying Forms. Aphasiology, 24(10), 1242-1266.

https://doi.org/10.1080/02687030903474255

Tomasello, M. (2003). Constructing a language: A usage-based theory of language acquisition. 
Cambridge, Mass.; London: Harvard University Press.

Tyler, L. K., Moss, H. E., Patterson, K., \& Hodges, J. (1997). The Gradual Deterioration of Syntax and Semantics in a Patient with Progressive Aphasia. Brain and Language, 56(3), 426-476. https://doi.org/10.1006/brln.1997.1857

Varley, R. (2011). Rethinking aphasia therapy: A neuroscience perspective. International Journal of Speech-Language Pathology, 13(1), 11-20. https://doi.org/10.3109/17549507.2010.497561

Varley, R., Cowell, P. E., Dyson, L., Inglis, L., Roper, A., \& Whiteside, S. P. (2016). Self-Administered Computer Therapy for Apraxia of Speech: Two-Period Randomized Control Trial with Crossover. Stroke, 47(3), 822-828. https://doi.org/10.1161/STROKEAHA.115.011939

Varley, R., \& Whiteside, S. P. (2001). What is the underlying impairment in acquired apraxia of speech? Aphasiology, 15(1), 39-49. https://doi.org/10.1080/02687040042000115

Veale, J. F. (2014). Edinburgh Handedness Inventory - Short Form: A revised version based on confirmatory factor analysis. Laterality, 19(2), 164-177. https://doi.org/10.1080/1357650X.2013.783045

Webster, J., Whitworth, A., \& Morris, J. (2015). Is it time to stop "fishing"? A review of generalisation following aphasia intervention. Aphasiology, 29(11), 1240-1264. https://doi.org/10.1080/02687038.2015.1027169

Wechsler, D. (2011). Wechsler Abbreviated Scale of Intelligence - Second Edition (WASI-II). San Antonio, TX: Psychological Corporation.

Whiteside, S. P., Inglis, A. L., Dyson, L., Roper, A., Harbottle, A., Ryder, J., ... Varley, R. A. (2012). Error reduction therapy in reducing struggle and grope behaviours in apraxia of speech. Neuropsychological Rehabilitation, 22(2), 267-294. https://doi.org/10.1080/09602011.2011.639614

Zimmerer, V. C., Newman, L., Thomson, R., Coleman, M., \& Varley, R. (2018). Automated analysis of language production in aphasia and right hemisphere damage: frequency and collocation strength. Aphasiology, 32(11), 1267-1283. https://doi.org/10.1080/02687038.2018.1497138

Zimmerer, V. C., Wibrow, M., \& Varley, R. A. (2016). Formulaic language in people with probable Alzheimer's Disease: a frequency-based approach. Journal of Alzheimer's Disease, 53(3), 11451160. https://doi.org/10.3233/JAD-160099 